Check for updates

Cite this: RSC Adv., 2019, 9, 8480

Received 29th January 2019

Accepted 7th March 2019

DOI: $10.1039 / c 9 r a 00789 j$

rsc.li/rsc-advances

\section{Novel in situ synthesis of copper nanoparticles supported on reduced graphene oxide and its application as a new catalyst for the decomposition of composite solid propellants $\uparrow$}

\author{
Paulina L. Ríos, ${ }^{a}$ Paula Povea, ${ }^{b}$ Christopher Cerda-Cavieres, ${ }^{a} J u a n$ L. Arroyo, ${ }^{b}$ \\ Cesar Morales-Verdejo, ${ }^{a}$ Gabriel Abarca (D) a and María B. Camarada (D) *a
}

\begin{abstract}
The catalytic activity of graphene oxide (GO), reduced graphene oxide (rGO), copper nanoparticles (CuNP) and rGO supported copper nanoparticles (rGO|CuNP) was investigated for the thermal decomposition of ammonium perchlorate (AP). GO was synthesized using a methodology based on hydrophilic oxidation, while an environmentally friendly and non-toxic reducing agent, L-ascorbic acid, was applied for the in situ reduction of copper and GO. The supporting rGO reduced the mean size of the copper nanoparticles from approximately 6 to $2 \AA$ due to the presence of stabilizing functional groups on the graphitic structure. Theoretical studies through Density Functional Theory revealed the important role of the epoxy and carbonyl groups of rGO on the stabilization of copper. The thermal decomposition process was studied based on DSC and TGA. GO, and rGO did not show a significant catalytic influence in the decomposition of AP. CuNP reduced the decomposition temperature of AP in greater magnitude than rGO|CuNP however, the synergistic effect of the rGO and CuNP increased the energy release significantly.
\end{abstract}

\section{Introduction}

Among the different fuels used to propel a rocket, those based on heterogeneous solid ingredients are commonly used in modern vehicles due to their higher safety, reliability, and stability in comparison to liquid systems. ${ }^{\mathbf{1}, 2}$ Composite solid propellants (CSP) are based on three main compounds: a binder, an oxygen-rich solid oxidizer, and a combustible metal additive like aluminum. ${ }^{3}$ The oxidizer is the source of oxygen and the major component of the composite solid propellant. Ammonium perchlorate (AP) is the most common oxidizer due to its excellent capabilities. ${ }^{4}$ The general performance of the CSP mainly depends on the decomposition temperature of AP. The lower the decomposition temperature of AP, the higher will be the combustion rate. The final decomposition temperature and the burn rate of composite solid propellants can be easily improved by adding a burn rate catalyst. ${ }^{5}$ Currently, burn rate catalysts mainly include transition metal oxides, metal nanoparticles, and ferrocene derivatives. Unfortunately, they do not accelerate the decomposition process as required.

${ }^{a}$ Centro de Nanotecnología Aplicada, Facultad de Ciencias, Universidad Mayor, Santiago, Chile. E-mail: maria.camarada@umayor.cl

${ }^{b}$ Laboratorio de Materiales Energéticos, Instituto de Investigaciones y Control del Ejército de Chile (IDIC), Av. Pedro Montt 2136, Santiago, Chile

$\dagger$ Electronic supplementary information (ESI) available. See DOI: $10.1039 / \mathrm{c} 9 \mathrm{ra} 00789 \mathrm{j}$
Metal nanoparticles have larger surface area compared to micron size catalysts and thus, exhibit higher surface energy and can enhance the burn rate and thermal decomposition of AP effectively. ${ }^{6-9}$ Because of their small size and high-surface-tovolume ratio, pure transition metal nanoparticles are very sensitive to oxygen and have led to more rapid thermal decomposition of AP. ${ }^{\mathbf{1 0 , 1 1}} \mathrm{Liu}$ and coworkers investigated the effect of metal nano- and micro-particles on thermal decomposition of AP. The nanometric metal powders increased the burning heat and rate decomposition of AP. The addition of $\mathrm{Cu}$, $\mathrm{Ni}$ and $\mathrm{Al} \mathrm{NPs} \mathrm{(5 \%} \mathrm{wt.)} \mathrm{lowered} \mathrm{the} \mathrm{high-temperature} \mathrm{decom-}$ position (HTD) peak of AP by $130{ }^{\circ} \mathrm{C}, 113{ }^{\circ} \mathrm{C}$ and $52{ }^{\circ} \mathrm{C}$ respectively, more than the micrometric particles. Copper nanoparticles (CuNP) were the best catalyst for promoting the decomposition of AP. ${ }^{12}$ Among the studies related to copper nanoparticles, most of them are directed to evaluate the activity of copper oxide. Only a few of them are related to the evaluation of neutral copper nanoparticles. ${ }^{12,13}$

Patil and coworkers, ${ }^{6}$ described the use of nano-copper oxide and nano-copper chromite as burning rate catalysts of AP, the latter exhibiting better results, lowering the HTD by $118{ }^{\circ} \mathrm{C}$ at $2 \%$ wt. Mahinroosta reported the application of $40 \mathrm{~nm}$ commercial CuO nanoparticles, diminishing the HTD of AP by $108{ }^{\circ} \mathrm{C}$ with $3 \%$ wt. nanoparticles. ${ }^{\mathbf{1 4}}$ More recently, Sharma et al. ${ }^{15}$ reported the green synthesis of $\mathrm{CuO}$ by using the extract of the leaves of a plant. The 30-40 nm nanoparticles decreased 
the HTD of AP by $115{ }^{\circ} \mathrm{C}$ at $1 \%$ wt. Among the studies related to neutral metallic copper nanoparticles, Dubey et al. studied the effect of $20 \mathrm{~nm}$ CuNP on the thermal decomposition of AP. Results indicated that nanoscale copper lowers the activation energy for thermal decomposition of AP and produces a $74{ }^{\circ} \mathrm{C}$ decay of the HTD of AP with $1 \%$ wt. catalyst. $^{13}$

In search of new catalysts, Sabourin et al. ${ }^{16}$ evaluated graphene (GR) as a catalyst of the combustion of composite solid propellants. GR, which has a high thermal ${ }^{17}$ and electrical conductivity, ${ }^{18,19}$ influenced the propellant burn rate through the acceleration of the electron transfers involved in the decomposition of AP. GR not only catalyzes the combustion reactions, but also participates energetically as a fuel, being consumed without producing residual particulates. Graphene oxide (GO), which consists of defect-rich graphene and residual chemisorbed oxygen-containing moieties, ${ }^{20}$ offers a less expensive alternative for the obtention of a graphene derivative: reduced graphene oxide (rGO), which may be equally effective for propellant applications as GR. However, the reduction of GO to produce rGO in large scale involves not only the removal of oxygen moieties but also the recovery or repair of the graphitic network. ${ }^{21,22}$

By now, there are several methods to reduce GO that affect the final performance of the material. ${ }^{23}$ Among them, the chemical approximation is simpler, cheaper and faster than physical paths, giving high yields with controllable properties. Several reducing agents have been assayed to resemble the pristine graphene structure. The most extended one is hydrazine hydrate due to its ability to produce rGO sheets with high electrical conductivity. ${ }^{24}$ However, this substance is very toxic to the environment and the living organisms. As an alternative, green reduction mechanisms have been explored by using amino acids, ${ }^{25}$ organic acids, ${ }^{26}$ and sugars, ${ }^{27}$ among other chemicals. The first known environmental friendly reducing agent of GO was ascorbic acid. Currently, it is the best non-toxic alternative, giving similar final characteristics to rGO compared to the hydrazine-reduced GO. ${ }^{28}$

Graphene and its derivatives can also act as support and stabilizer of metal nanoparticles ${ }^{29,30}$ like copper. In 2008, Luechinger and coworkers ${ }^{31}$ reported a bottom-up approach to synthesize copper nanoparticles coated with a protective shell of graphene to be used as low-cost printable ink for electronics. Zhang et $a .^{32}$ produced rGO|CuNP composites by a chemical reduction method with $\mathrm{NaBH}_{4}$, with application in surfaceenhanced Raman scattering. In 2014 two groups reported the synthesis of rGO|CuNP with application in chemical transformation reactions. ${ }^{33,34}$ Only one of the studies corresponded to the in situ generation of the stabilized nanoparticles, however, it implied a non-green chemical reductant. ${ }^{34}$

By now, a few studies are referred to the supported synthesis of copper nanoparticles with direct use as an accelerator of the decomposition of AP. During 2017 two groups described the application of $\mathrm{CuO}$ supported on graphene derivatives as burn rate catalyst of AP. Hosseini et al. enhanced the catalytic performance, diminishing $111^{\circ} \mathrm{C}$ the HTD by the application of $4 \%$ wt. of copper oxide nanoparticles supported on nitrogendoped graphene, ${ }^{35}$ while Paulose and coworkers introduced a nanocomposite of functionalized white graphene and copper oxide, achieving a reduction of $50{ }^{\circ} \mathrm{C}$ of the $\mathrm{HTD}$ with $3 \%$ wt. of the catalyst. ${ }^{36}$

To our knowledge, among literature, there are no reports about the specific use of neutral copper nanoparticles supported on reduced graphene oxide ( $\mathrm{rGO} \mid \mathrm{CuNP}$ ) as burning rate catalyst of composite solid propellants, produced in situ by a green chemical reductant as ascorbic acid. Therefore, in this work, we report the first in situ synthesis and characterization of copper nanoparticles supported on rGO and their further application as catalysts for the decomposition of AP. Although CuNP exhibited better performance as catalyst than rGO|CuNP, the latter increased importantly the energy release. The interaction between rGO and copper was also analyzed through $a b$ initio calculations, revealing the vital role of the functional groups for the stabilization of the metal.

\section{Experimental details}

\subsection{Reagents and characterization methods}

Reagents were of analytical grade or the highest commercially available purity and were used as received. Graphite, $\mathrm{KMnO}_{4}$, $\mathrm{H}_{2} \mathrm{SO}_{4}, \mathrm{H}_{3} \mathrm{PO}_{4}, \mathrm{H}_{2} \mathrm{O}_{2}, \mathrm{HCl}$, ethanol, L-ascorbic acid, $\mathrm{NH}_{3}$, and copper dichloride dihydrate were purchased from Merck. Aqueous solutions were prepared with in-house ultrapure water of resistivity not less than $18 \mathrm{M} \Omega \mathrm{cm}$ (Milli-Q, USA). Fourier transform infrared spectroscopy (FT-IR) spectra were measured using a Perkin Elmer Spectrum two UATR spectrometer in the frequency range $500-4000 \mathrm{~cm}^{-1}$. UV-visible spectra were recorded with a Merck Spectroquant Prove 300 spectrophotometer. All measurements were taken at room temperature. Differential scanning calorimetry (DSC) analysis were performed on an $822 \mathrm{e}$ Mettler Toledo instrument at a heating rate of $5{ }^{\circ} \mathrm{C} \mathrm{min}{ }^{-1}$ under a nitrogen blanket in the range of $140-500{ }^{\circ} \mathrm{C}$. Scanning transmission electron microscopy (STEM) and high-resolution transmission electron microscopy (HRTEM) analyses were performed with a FEI Titan Themis 80/300 microscopy at LNNano (LNLS, Brazil), operated at $300 \mathrm{kV}$. High Z-contrast images were acquired through STEM using a high-angle annular dark field detector (HAADF) and a semiconvergence angle of $27.4 \mathrm{mrad}$. The typical resolution was greater than $0.01 \mathrm{~nm}$. The samples were prepared by directly applying the CuNP powder onto standard TEM grids. TEM images were collected on a Hitachi HT7700 equipment. The catalytic performance of the compounds for the thermal decomposition of ammonium perchlorate (AP) was investigated by adding the catalysts to AP (20 $\mu \mathrm{m}$ size) in 1, 3 and $5 \% \mathrm{wt}$.

\subsection{Synthesis and characterization of graphene oxide (GO) and reduced graphene oxide (rGO)}

Slightly modified Marcano' method was used to produce GO, ${ }^{37}$ which provides a greater quantity of hydrophilic oxidized graphite material in comparison to the traditional Hummers method, ${ }^{38}$ with higher yield and simpler protocol. Graphite powder $(3.0 \mathrm{~g})$ and $\mathrm{KMnO}_{4}(18.0 \mathrm{~g})$ was added to a mixture of $\mathrm{H}_{2} \mathrm{SO}_{4} / \mathrm{H}_{3} \mathrm{PO}_{4}(360 \mathrm{~mL} / 40 \mathrm{~mL})$, and the reaction occurred at 
$50{ }^{\circ} \mathrm{C}$ for $12 \mathrm{~h}$. The mixture was cooled to room temperature and poured onto ice $(\approx 400 \mathrm{~mL})$ with $30 \% \mathrm{H}_{2} \mathrm{O}_{2}(3 \mathrm{~mL})$. The mixture was centrifuged (6000 rpm, $2 \mathrm{~h}$ ) and the supernatant decanted away. The solid was washed with deionized water $(200 \mathrm{~mL}), 30 \%$ $\mathrm{HCl}(200 \mathrm{~mL})$ and ethanol $(200 \mathrm{~mL}$, twice) and centrifuged in each wash step. Then, the solid was coagulated with ether, filtered $(2 \mu \mathrm{m})$ and dried in a vacuum oven at $30{ }^{\circ} \mathrm{C}$.

Chemical conversion of GO to reduced graphene oxide (rGO) was completed by a green reductant, according to a reported method: ${ }^{39} 100 \mathrm{mg}$ of GO were dispersed in $1000 \mathrm{~mL}$ of milli-Q water. Then $1 \mathrm{~g}$ of L-ascorbic acid was added, and the mixture was stirred and heated at $65^{\circ} \mathrm{C}$ for $1 \mathrm{~h}$. The $\mathrm{pH}$ value was kept at 10 by adding $\mathrm{NH}_{3}$ dissolution. Finally, the rGO was collected by filtration. The resulting product, a black powder, was washed several times with milli-Q water and was dried in a vacuum oven at $30{ }^{\circ} \mathrm{C}$.

\subsection{Synthesis and characterization of copper nanoparticles (CuNP) and copper nanoparticles supported on reduced graphene oxide (rGO|CuNP)}

Xiong' method with some modifications was used in the production of copper nanoparticles (CuNP). ${ }^{40} \mathrm{CuCl}_{2} \cdot 2 \mathrm{H}_{2} \mathrm{O}(25$ $\mathrm{mL}, 0.2 \mathrm{M}$ ) was heated to $80^{\circ} \mathrm{C}$ while stirring. L-ascorbic acid (25 $\mathrm{mL}, 0.4 \mathrm{M}$ ) was added dropwise, and the mixture was kept in heating $\left(80^{\circ} \mathrm{C}\right)$ stirring overnight $(12 \mathrm{~h})$. The mixture was cooled to room temperature, centrifuged (8000 rpm, $15 \mathrm{~min})$ and washed with milli-Q water. The supernatant was diluted to $50 \mathrm{~mL}$ in a volumetric flask. The CuNP $(0.1 \mathrm{M})$ was kept up to 2 months.

In the case of rGO|CuNP, a novel synthesis was developed to produce the in situ reduction of copper and graphene oxide: previously synthesized GO $(127.1 \mathrm{mg})$ was dispersed in $\mathrm{CuCl}_{2}$ dissolution $(10 \mathrm{~mL}, 0.2 \mathrm{M})$ and then put in stirring heat $\left(80^{\circ} \mathrm{C}\right)$. An L-ascorbic acid dissolution $(10 \mathrm{~mL}, 1.23 \mathrm{M})$ was added dropwise, and the mixture was kept in stirring heat $\left(80^{\circ} \mathrm{C}\right)$ for 12 hours. The $\mathrm{pH}$ was adjusted to 10 by adding $\mathrm{NH}_{3}$ dissolution. Nanoparticles were kept in solution. TEM, UV-vis, and FT-IR analysis were performed.

\subsection{Computational details}

Theoretical approximations explored the nanoarchitecture of rGO and its interaction with CuNP. Because of the size of the rGO system, it was represented by a $2 \times 2 \mathrm{~nm}^{2}$ structure. The structure of rGO was built according to a previous study of the reduction of GO by ascorbic acid. ${ }^{39}$ The GO structure presents epoxy, alkoxy, hydroxyl, carbonyl, and carboxyl groups. Epoxy and hydroxyl groups are mainly located on the surface of the graphitic network and are the most labile moieties. After the reduction process, these groups are partly removed. The chemical reduction of GO does not eliminate completely the surface and the edge functionalities, ${ }^{\mathbf{4 1}}$ which can stabilize metal ions. The geometry of rGO was fully optimized at the density functional theory (DFT) level using the Gaussian16 computational package. ${ }^{42}$ Copper nanoparticles were represented by an eight-atom cluster $\left(\mathrm{Cu}_{8}\right)$. With the aim of studying the most stable site of coordination between copper nanoparticles and
rGO, full geometry optimizations of complexation reactions were performed using Becke's three parameters nonlocal hybrid exchange potential with nonlocal correlation functional of Lee, Yang, and Parr (B3LYP) ${ }^{\mathbf{4 3 - 4 5}}$ without any symmetry restriction. The triple- $\zeta$ 6-311G basis set was set for light atoms $(\mathrm{C}, \mathrm{H}, \mathrm{O})$ along with a relativistic effective core potential basis set with pseudopotentials for copper atoms, LANL2DZ. ${ }^{46}$ A tight SCF convergence criterion $\left(10^{-8}\right.$ a.u.) was used in all calculations. As implemented in Gaussian16, the charge distribution of intermolecular interactions was calculated using natural population analysis (NPA) method. ${ }^{47}$ Interaction energy $\left(E_{\text {int }}\right)$ was defined as the energy difference between the complex and energies of constituent monomers and was calculated using the following expression: $E_{\mathrm{int}}=E_{\mathrm{rGO}-\mathrm{Cu}_{8}}-E_{\mathrm{rGO}\left(\mathrm{rGO}_{-} \mathrm{Cu}_{8}\right)}-E_{\mathrm{Cu}_{8}(\mathrm{rGO}-\mathrm{Cu})}$. The computation of this quantity with finite basis sets introduces error known as basis set superposition error (BSSE) because different numbers of basic functions were used to describe the complex and monomers for the same basis set. BSSE corrected interaction energies were computed using Boys-Bernardi counterpoise correction scheme. ${ }^{48}$ The effect on the conductivity of the coordination of copper to reduced graphene oxide was estimated by the energy of band gap $\left(E_{\mathrm{BG}}\right)$, which corresponds to the energy difference between the highest occupied molecular orbital (HOMO) and the lowest unoccupied molecular orbital (LUMO).

\section{Results and discussion}

\subsection{Synthesis of GO and rGO}

The FT-IR spectra of graphite and GO are shown in Fig. S1a. $\dagger$ The spectrum of GO revealed oxygen functionalities at 3280 (O$\mathrm{H}$ stretching vibrations), 1720 ( $\mathrm{C}=\mathrm{O}$ stretching vibrations), and $1230 \mathrm{~cm}^{-1}$ (C-O stretching vibrations), characteristic of the interruptions of the graphene lattice by oxygen-containing groups like alcohols, epoxides, and carbonyls. At $1615 \mathrm{~cm}^{-1}$ appeared $\mathrm{C}=\mathrm{C}$ skeletal vibrations from un-oxidized graphitic diamonds. The spectrum of pure graphite had absorption bands at 3440 and $1040 \mathrm{~cm}^{-1}$ corresponding to the $\mathrm{O}-\mathrm{H}$ and $\mathrm{C}-\mathrm{O}$ stretching vibrations respectively. ${ }^{49}$ The absorption peak at $1640 \mathrm{~cm}^{-1}$ was attributed to the skeletal vibrations of $\mathrm{C}=\mathrm{C}^{50}$ The GO spectrum is entirely different from the graphite spectrum, and contains fewer oxygen-containing functional groups, confirming the successful oxidation of the graphite. In the case of $\mathrm{rGO}$, the $\mathrm{O}-\mathrm{H}$ stretching vibrations were significantly reduced in comparison with GO as a result of the deoxygenation process and the removal of most oxygen functionalities.

UV-visible spectra of the samples were also measured in water $\left(0.07 \mathrm{mg} \mathrm{mL}^{-1}\right)$. As Fig. $\mathrm{S} 1 \mathrm{~b} \dagger$ shows, in the case of GO two bands were detected and assigned to the $\pi \rightarrow \pi^{*}$ transition (230 $\mathrm{nm}$ ) of the aromatic rings and $\mathrm{n} \rightarrow \pi^{*}$ of the carbonyl groups at approximately $318 \mathrm{~nm} .{ }^{51}$ After reduction of the functional groups with ascorbic acid, rGO exhibits its characteristic band at $270 \mathrm{~nm}$. The bathochromic shifting of the bands is related to the increase of electronic conjugation after reduction. ${ }^{52}$ The higher conjugation degree, the less energy needs to be applied to produce the electronic transition, and thus, the peak wavelength moves to longer values, associated with less energy. This 
fact indicates rGO has more aromatic rings or aromatic domains than GO.

Transmission electron microscopy images of GO and rGO were taken. As Fig. 1a shows, the GO synthesis produced large flakes with wavy edges with multiple layers thick.

After its reduction with ascorbic acid to produce $\mathrm{rGO}$, the images (Fig. 1b) indicated the presence of more regular carbon framework, where the atomic columns of the carbon are visible with a spacing of approximately $3 \AA$, shown in the inset of the Fig. 1b.

\subsection{Synthesis of CuNP and rGO|CuNP}

Particles at the nanoscale have unique optical characteristics. In this sense, UV-vis spectroscopy has proved to be very useful for the characterization of nanoparticles, due to the relation of the position and shape of the peak with the size of the particles. Copper nanoparticles were synthesized following the procedure of Xiong et al. ${ }^{40} \mathrm{UV}$-vis spectroscopy was performed to reagent and product samples.

Fig. 2a shows the $\mathrm{CuCl}_{2}$ spectrum with a peak at $230 \mathrm{~nm}(\mathrm{Cu}-$ $\mathrm{Cu}$ interaction) and a broad peak at $810 \mathrm{~nm}$, assigned to the $\mathrm{Cu}-$ $\mathrm{H}_{2} \mathrm{O}$ interaction. ${ }^{53}$ The CuNP curve at $\mathrm{pH} 1$ (resulting from the synthesis) shows shoulder type peaks at 200, 242 and $300 \mathrm{~nm}$. Additionally, a close up to the $300-600 \mathrm{~nm}$ region, exhibits a third peak at $390 \mathrm{~nm}$, caused by the change in the surface plasmon resonance of CuNP and indicates the formation of copper nanoparticles. The spectrum of $\mathrm{rGO} \mid \mathrm{CuNP}$ exhibits a peak a $270 \mathrm{~nm}$, which is associated to rGO and the characteristic peak of the copper nanoparticles at $200 \mathrm{~nm}$, confirming the effective reduction of $\mathrm{GO}$ and copper chloride. The rGO|CuNP sample was also characterized by FT-IR spectroscopy (Fig. 2b). In comparison to $\mathrm{GO}$, the characteristic carbonyl $\mathrm{C}=\mathrm{O}$ band disappeared, while the $\mathrm{C}-\mathrm{O}$ was also not registered. The
$\mathrm{O}-\mathrm{H}$ band also diminished its intensity. The new peaks at the $\mathrm{rGO} \mid \mathrm{CuNP}$ sample were associated with the ascorbic acid. All this evidence confirms the effective reduction of GO to rGO.

The morphology and size distribution of the CuNP sample was assessed by Transmission Electron Microscopy (TEM). As Fig. 3a shows, the particles are in general spherical. The mean particle diameter $(6 \mathrm{~nm})$, was determined by the histogram of the size distribution, also shown in Fig. $3 \mathrm{a}$.

The presence of CuNP was observed by HAADF-STEM (Fig. 4a). The mean $\mathrm{Cu}$ particle size is $4.8 \pm 1.2 \mathrm{~nm}$, being calculated over a range of 100 particles (Fig. S2 $\dagger$ ). The d-spacing of $0.212 \AA$ is in a good agreement with the (111) plane of the fcc phase of $\mathrm{Cu}(0)$. The presence of $\mathrm{Cu}$ was also probed by a single particle STEM-EDS profile (Fig. 4b), where the identification of the $\mathrm{Cu} \mathrm{K} \alpha$ and $\mathrm{Cu} \mathrm{K} \beta$ evidence the presence of copper.

The rGO|CuNP sample was also examined by TEM. Fig. $3 \mathrm{~b}$ shows the histogram of the size distribution of the copper nanoparticles, with a mean size of $2 \mathrm{~nm}$. The decrease of the CuNP size is related to the stabilizing effect of the functional groups of rGO. GO has different functionalities like epoxy, alkoxy, hydroxyl, carboxyl, and carbonyl groups. Epoxy and hydroxyl groups are mainly located on the surface of the conjugated carbon skeleton and are partially removed after the chemical reduction with ascorbic acid..$^{41}$ Nevertheless, the edge functionalities, such as $-\mathrm{COOH},-\mathrm{C}=\mathrm{O}$ and $-\mathrm{OH}$, remain in position, being potential sites for the stabilization of metal nanoparticles. The effect of each group on the coordination of rGO to copper nanoparticles was explored by DFT, as discussed in the following section.

\subsection{Computational description of $\mathrm{rGO} \mid \mathrm{CuNP}$}

The rGO structure was designed according to the results of De Silva $e t$ al. ${ }^{39}$ who employed ascorbic acid for the reduction of
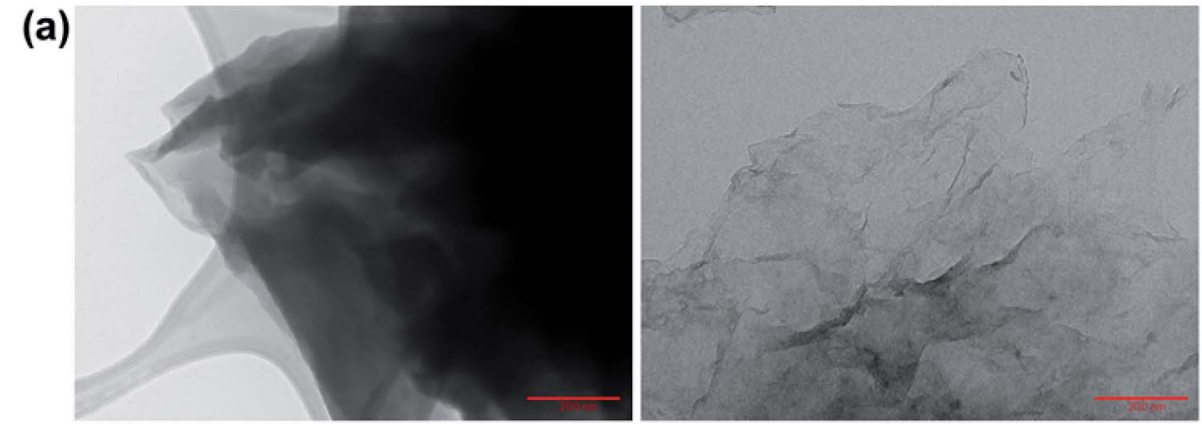

(b)
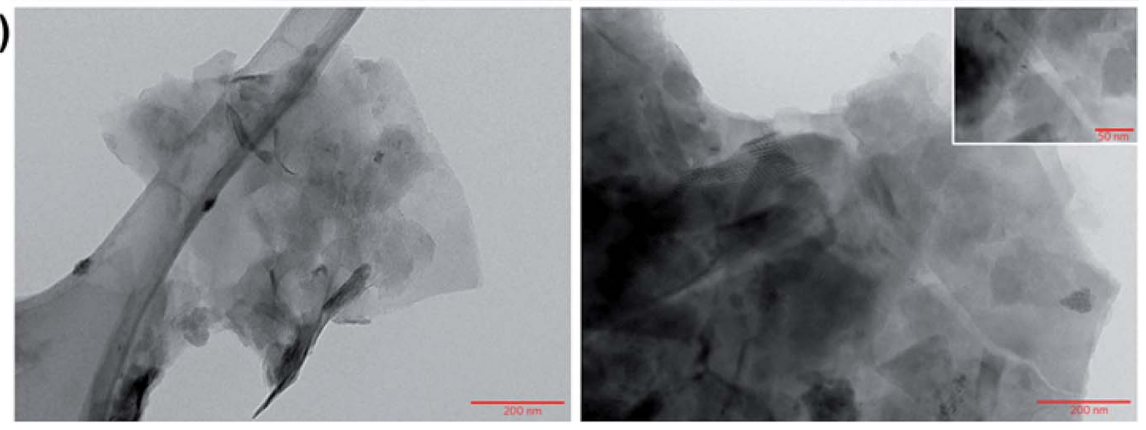

Fig. 1 TEM images of (a) GO and (b) rGO. TEM grids with lacey carbon support films (Ted Pella Inc.) were used to prepare the samples. 
(a)

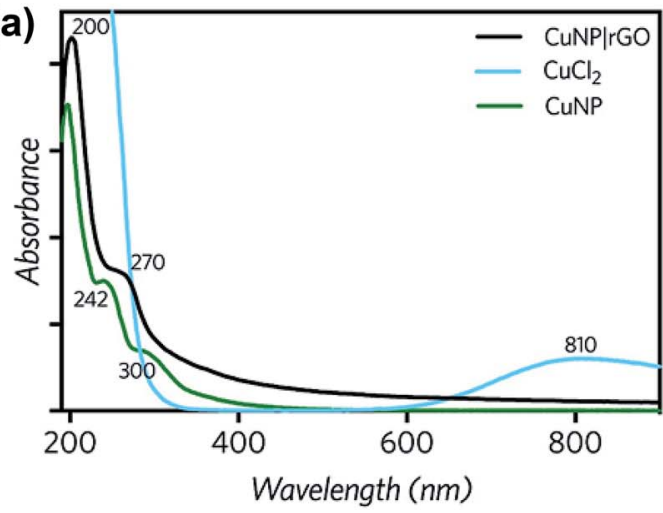

(b)

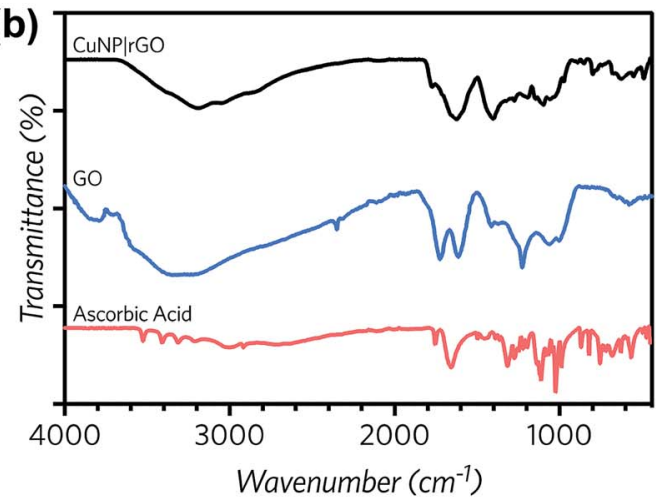

Fig. 2 (a) UV-vis spectra of $\mathrm{CuCl}_{2} 0.2 \mathrm{mM}$ and the synthesized CuNP and rGO|CuNP. (b) FT-IR spectra of rGO|CuNP, GO and ascorbic acid.

GO. The rGO was fully optimized at B3LYP/6-311G level and used as reference for the calculation of BSSE-corrected interaction energy $\left(E_{\text {int }}\right)$. The following functional groups were considered to analyze the energetic interaction with the eightatom copper cluster $\left(\mathrm{Cu}_{8}\right)$ : carboxylic acid, epoxy, alcohol and carbonyl. The starting geometries of $\mathrm{rGO} \mid \mathrm{Cu}_{8}$ complexes were generated by placing the $\mathrm{Cu}_{8}$ cluster near the electron-rich sites of the rGO structure. Five different coordination sites were considered, as Fig. 5a shows: $\mathrm{Cu}_{8}$ interacting with (I) the carbonyl group $(\mathrm{C}=\mathrm{O})$, (II) the carboxylic acid group (-COOH), (III) the hydroxyl group (-OH) at edges of the rGO layer, and (IV) the epoxy groups $(-\mathrm{O}-)$ and $(\mathrm{V})$ the hydroxyl $(-\mathrm{OH})$ on the surface of the rGO layer.

Three initial conformations were tested at each coordination site to search for alternative local minima. Table 1 summarizes the most stable $E_{\text {int }}$ at each coordination site, distances between the $\mathrm{Cu}_{8}$ cluster and the oxygen anchor atom, the charge for the optimized complexes and the energy band gap.

The $E_{\text {int }}$ analysis indicates that the most stable coordination site is number (IV), with interaction at the epoxy site (Fig. 5b), then complexes (I) and (V), with coordination to the carbonyl and $-\mathrm{OH}$ on the surface of $\mathrm{rGO}$, respectively. Fig. $\mathrm{S} 3 \uparrow$ shows the optimized geometry of all complexes, in which bond lengths and NBO charges at selected atomic sites are depicted. Complex (IV) is $11.3 \mathrm{kcal} \mathrm{mol}^{-1}$ more stable than system (I). Moreover, the coordination of the copper cluster to rGO at the epoxy group presented the shorter anchor bond distance of all the set, associated with a stronger interaction and stability.

The total NPA charge of the $\mathrm{Cu}_{8}$ cluster $\left(\Delta q_{\text {cluster }}\right)$ was also analyzed. In the case of the most stable complexes, (IV) and (I), there was a charge transfer from the copper cluster to the rGO, while in the rest of the systems migration of the electron density (a)
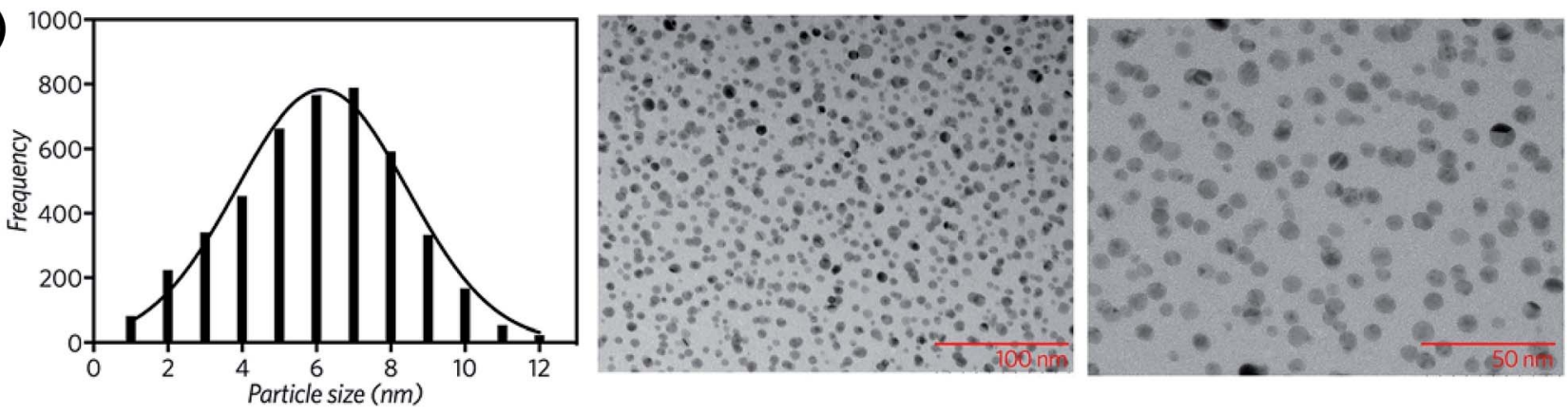

(b)

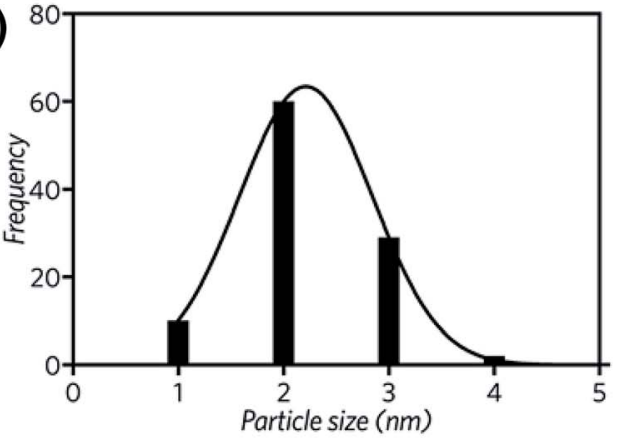

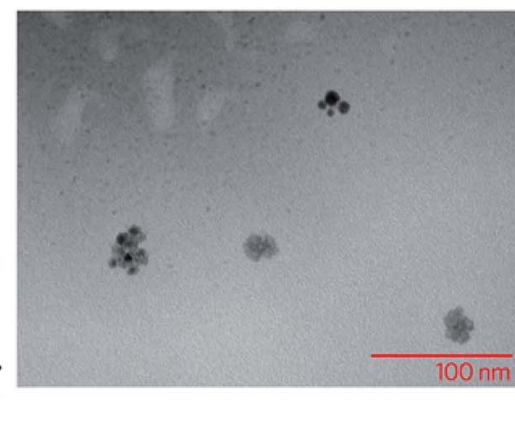

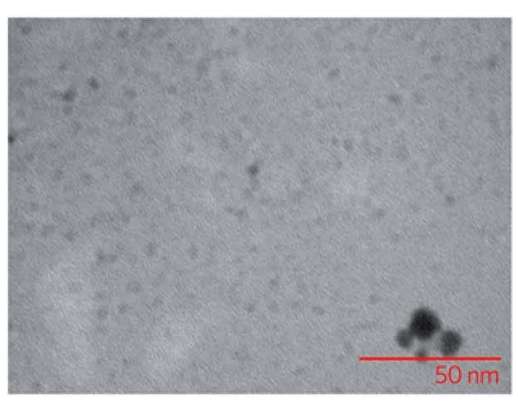

Fig. 3 Particle size distribution and TEM images of the synthesized (a) copper nanoparticles and (b) copper nanoparticles supported on rGO. 
(a)

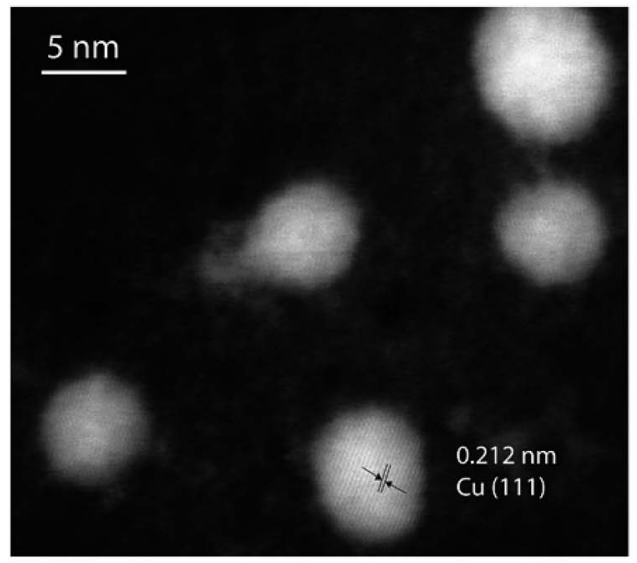

(b)

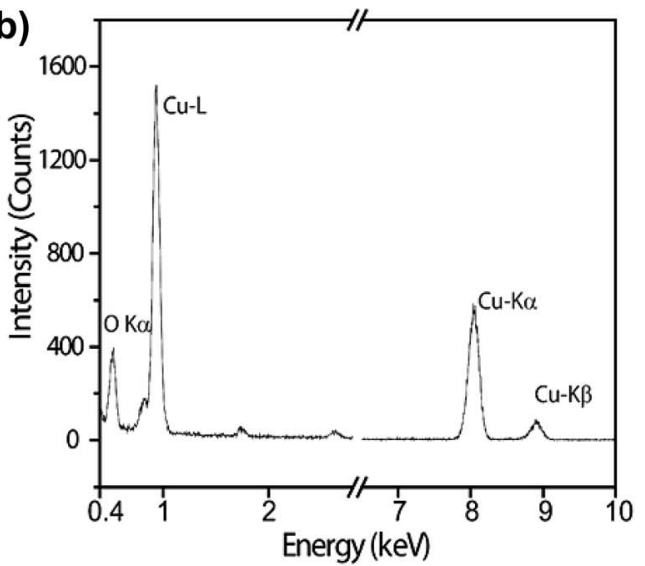

Fig. 4 (a) Selected HAADF-STEM image of CUNP and (b) STEM-EDS profile of CuNP.

occurred in the opposite direction, i.e., from the rGO ligand to the metal cluster. The electron transfer had a direct impact on the energy of the frontier orbitals of the systems. Complexes (IV) and (I), with higher interacting energy, presented lower band gap energies $\left(E_{\mathrm{BG}}\right)$, as depicted in Fig. $5 \mathrm{c}$. The presence of the $\mathrm{Cu}$ nanocluster coordinated to specific functional groups of the rGO structure, carbonyl and epoxy moieties, improved the delocalization of electronic charge around rGO rings. As can be seen from the electrostatic potential surface (EPS) of the optimized structure of rGO (Fig. S4 $\dagger$ ), most of the electronic density is located at the oxygen atoms belonging to the epoxy and carbonyl functional groups. This evidence indicates that the lone pairs of these oxygen atoms are highly available for the interaction with metal atoms. According to these results, CuNP will tend to grow close to -O- on the surface of the rGO layer or next to the carbonyl sites of the edges, rather than other functional groups of rGO.

\subsection{Application as propellant catalysts}

The use of nanostructured materials in ignition and combustion area is rapidly increasing. In this work, the synthesized systems were evaluated as catalysts for the decomposition of ammonium perchlorate, the main component of composite solid propellants. It is well known that the thermal decomposition of ammonium perchlorate (AP) involves electronic transfers and thus, the introduction of an electron transport agent could facilitate and accelerate the decomposition reaction of AP. It has been reported that graphene and graphene oxide derivatives are capable of constructing a conductive network that facilitates the heat and electron transfers during fuel decompositions, producing an improved energy release and decomposition of AP..$^{54,55}$

Dey et al. ${ }^{56}$ evaluated graphene as a catalyst of the decomposition of AP with a $5 \%$ wt., registering a first exothermic peak at $305{ }^{\circ} \mathrm{C}$ and diminishing the HTD process of AP by $6{ }^{\circ} \mathrm{C}$. Zhao and coworkers tested $\mathrm{GO}$ at $2 \%$ wt., observing two peaks for the low- and high-temperature decomposition at 345 and $430{ }^{\circ} \mathrm{C}$ respectively, ${ }^{57}$ diminishing the HTD by $3{ }^{\circ} \mathrm{C}$. A study published by Memon and coworkers ${ }^{54}$ reported that fast crash AP with hand-mixed GO decreased the second decomposition temperature of AP by $26{ }^{\circ} \mathrm{C}$, while fast crash AP and GO reduced the final decomposition temperature to $32{ }^{\circ} \mathrm{C}$.

GO, rGO, CuNP and rGO|CuNP were tested as catalysts for the decomposition of AP. As Fig. 6 shows, the AP curve has the

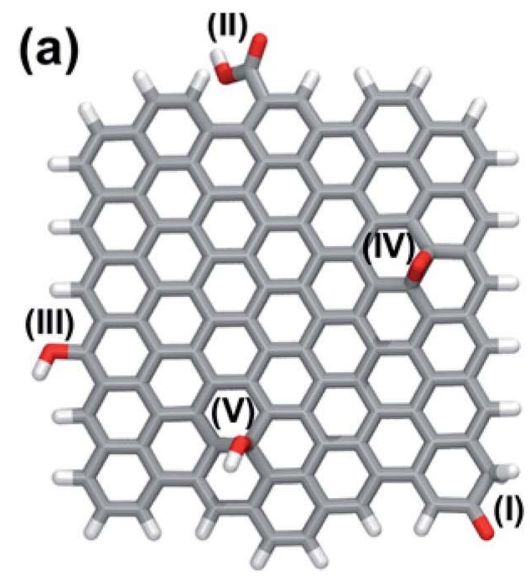

(b)

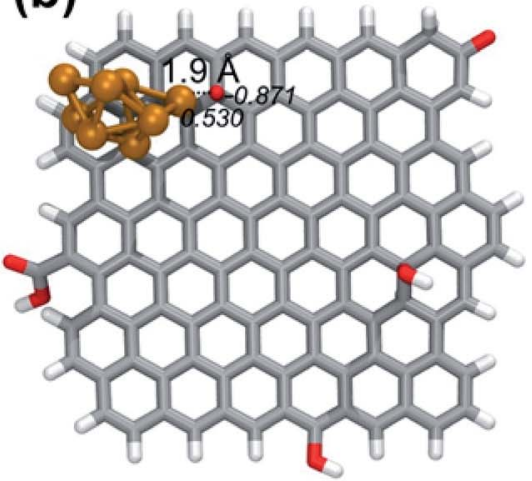

(c)

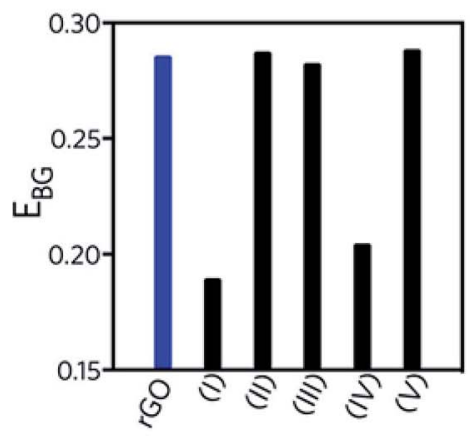

Fig. 5 (a) The optimized structure of $\mathrm{rGO}$ at the B3LYP/6-311G level showing the five possible coordination sites, (b) the most stable $r G O / C u_{8}$ complex (IV), NPA charges (a.u.) for selected atoms are displayed in italics, and bond lengths in $\AA$, and (c) theoretical energy band gap, calculated for each complex and rGO. 
Table $1 \mathrm{Cu}-\mathrm{O}$ anchor bond distances $d_{\mathrm{O}-\mathrm{Cu}}$ in $\AA$. NPA derived atomic charges of the anchor atom $q_{0}$, the bonded gold copper $q_{\mathrm{Cu}}$ and the total charge of the metal cluster $\Delta q_{\text {cluster }}$ in a.u. BSSE-corrected and -uncorrected (in parenthesis). Interaction energy $\left(E_{\text {int }}, \mathrm{kcal}_{\mathrm{mol}}^{-1}\right)$ for the studied complexes. Energy band gap $\left(E_{\mathrm{BG}}\right)$ in $\mathrm{eV}$

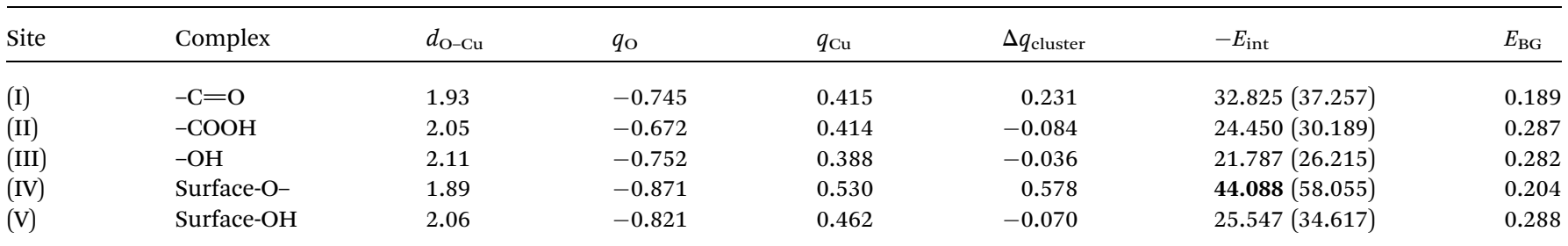

classical first endothermic peak corresponding to the crystal phase transition from orthorhombic to cubic phase (240-250 $\left.{ }^{\circ} \mathrm{C}\right) .{ }^{58}$ After that, the exothermic low-temperature decomposition (LTD) and sublimation take place at approx. $300^{\circ} \mathrm{C}$ with an electron transfer, which is the most accepted mechanism..$^{59}$ Finally, the high-temperature decomposition (HTD) step occurs at $415{ }^{\circ} \mathrm{C}$.

Differential scanning calorimetry curves (Fig. 6) were obtained for the synthesized compounds, mixed with AP in 1, 3 and $5 \%$ wt. In all cases, the catalysts did not affect the crystallographic transition process of AP. In the three GO-profiles (Fig. 6a), there is an initial endothermic peak at approximately $155{ }^{\circ} \mathrm{C}$, corresponding to the release of $\mathrm{CO}, \mathrm{CO}_{2}$, and steam from the most labile groups, i.e., from the surface oxygen functionalities on the graphitic net. ${ }^{24}$ The LTD of AP decreased by approximately $4{ }^{\circ} \mathrm{C}$ with the introduction of GO. The further decomposition of $\mathrm{GO}$ was detected between $380{ }^{\circ} \mathrm{C}$ and $433^{\circ} \mathrm{C}$, associated to the removal of the most stable oxygen functionalities located on the edges. ${ }^{60} \mathrm{GO}$ at $5 \%$ wt. diminished the HTD of AP by $12{ }^{\circ} \mathrm{C}$.

Fig. $6 \mathrm{~b}$ shows the DSC profiles of rGO. Unlike GO, endothermic processes $\left(\sim 150^{\circ} \mathrm{C}\right)$ associated with the combustion of oxygen moieties were not present, confirming the successful reduction of GO to $\mathrm{rGO}$ by the green agent ascorbic acid. Moreover, if the energy release is compared to GO, in the three different mass percentages, the released energy amount diminished, due to the lesser presence of functional groups. A second decomposition process occurred between 360 and $370^{\circ} \mathrm{C}$, related to the removal of residual oxygen functionalities. Considering $5 \%$ wt., the LTD decreased by $5{ }^{\circ} \mathrm{C}$ and $1{ }^{\circ} \mathrm{C}$ in the case of the HTD. In comparison to GO, rGO lowers both LTD and HTD and has no burning processes after the AP decomposition at approx. $415{ }^{\circ} \mathrm{C}$. Therefore, all the energy is released before the HTD of AP.
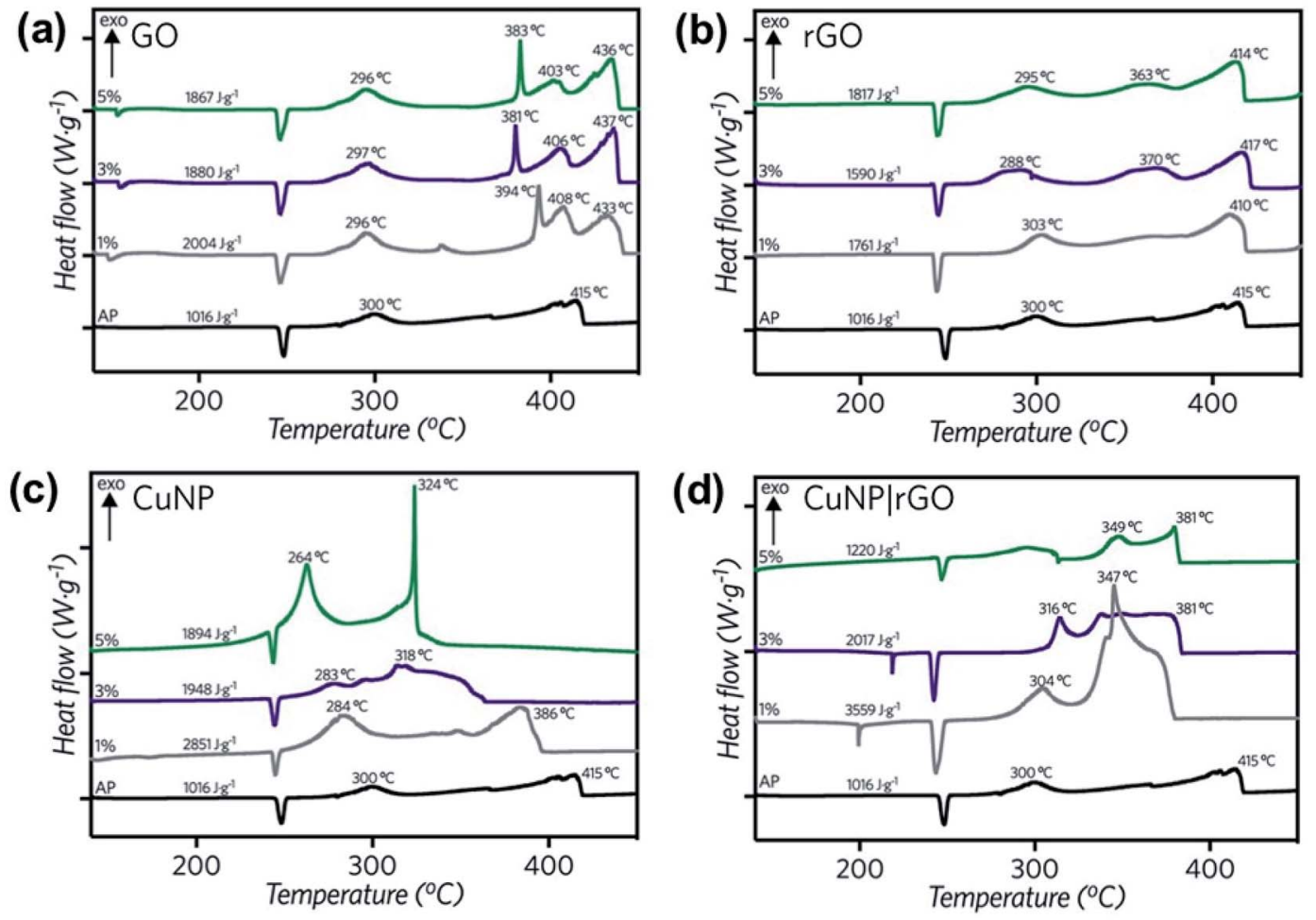

Fig. 6 DSC curves for the thermal decomposition of pure AP and AP with 1, 3 and 5\% wt. of (a) GO, (b) rGO, (c) CuNP and (d) rGO|CuNP. 
Copper nanoparticles (Fig. 6c) and rGO supported nanoparticles (Fig. 6d) were also tested as burning rate catalysts of AP. It has been previously described in the literature that AP is extremely sensitive to the action of additives. The application of nanoscale instead of microscale additives improves the catalytic action sharply. ${ }^{9}$ Most of the studies regarding copper nanoparticles are derived from CuO. Only a few of them report the use of copper nanoparticles. Sharma et al. ${ }^{15}$ lowered the HTD of AP by $115{ }^{\circ} \mathrm{C}$ at $1 \%$ wt. by applying $30-40 \mathrm{~nm}$ copper oxide nanoparticles, while Dubey et al. achieved a $74{ }^{\circ} \mathrm{C}$ decay of the HTD by employing $20 \mathrm{~nm}$ CuNP with $1 \%$ wt. catalyst. ${ }^{13}$ Liu and coworkers ${ }^{12}$ reduced the HTD by $130{ }^{\circ} \mathrm{C}$ using CuNP of $90 \mathrm{~nm}$, releasing $1.2 \mathrm{~kJ} \mathrm{~g}^{-1}, 2.75$ times higher than pure AP. In the present work, with $3 \%$ wt. of CuNP, the LTD was diminished by $17^{\circ} \mathrm{C}$, while the HTD decreased by $97^{\circ} \mathrm{C}$. Most importantly, at this concentration, the released energy almost duplicated the value of pure AP $\left(1.9 \mathrm{~kJ} \mathrm{~g}^{-1}\right)$. At $5 \%$ wt. the LTD decreased even more, by $36{ }^{\circ} \mathrm{C}$. The second decomposition process occurred in a single peak, $91{ }^{\circ} \mathrm{C}$ before pure $\mathrm{AP}$, suggesting a single decomposition process.

By now, there are a few studies applying graphene or graphene derivatives as support for the synthesis of neutral copper nanoparticles with direct use as accelerators of the decomposition of AP. Moreover, to our knowledge, there are no reports of the in situ production of copper nanoparticles supported on reduced graphene oxide by an environmental friendly reducing agent. Only copper oxide nanoparticles have been supported on graphene derivatives as burn rate catalyst of AP. Hosseini and coworkers $^{35}$ enhanced the catalytic performance of AP by the use of $4 \%$ wt. copper oxide nanoparticles supported on nitrogen-doped graphene. They diminished the HTD by $111^{\circ} \mathrm{C}$ and multiplied the energy release by 3.2 times. Paulose $e t$ al. ${ }^{36}$ achieved a $50{ }^{\circ} \mathrm{C}$ decrease of the HTD of AP, with $3 \%$ wt. of a nanocomposite of functionalized white graphene and copper oxide.

In the present work, the $\mathrm{rGO} \mid \mathrm{CuNP}$ DSC profile at 1 and $3 \%$ wt. exhibited a second endothermic peak, associated to the melting of the ascorbic acid. ${ }^{61}$ The composite at $1 \% \mathrm{wt}$. diminished the HTD of AP by $68{ }^{\circ} \mathrm{C}$. It is important to note that the CuNP assays contained the double amount of copper due to the presence of rGO in the composite sample. Although the HTD decreased by lesser amount in comparison to CuNP, the energy release increased 3.5 times, higher than the CuNP catalyst and the work reported by Hosseini and coworkers. ${ }^{35}$ The decrease in the decomposition temperature is close to the value reported by Dubey ${ }^{13}$ and coworkers with CuNP of $20 \mathrm{~nm}$ $\left(74{ }^{\circ} \mathrm{C}\right)$. As rGO has a low impact on the modification of the HTD of AP, the obtained result can be related to the effect of the copper nanoparticles on the decomposition mechanism. As Fig. 6b shows, rGO acts mainly as fuel during the combustion of AP. On the other hand, rGO|CuNP presented a higher effect on the decrease of the HTD compared to wellknown and typical AP catalysts, such as commercial copper chromite ${ }^{62}\left(43^{\circ} \mathrm{C}\right)$, ferric oxide ${ }^{63}\left(40^{\circ} \mathrm{C}\right)$ and ferrocene $e^{64}\left(58^{\circ} \mathrm{C}\right)$. In the literature, it is possible to find different copper-based catalysts with better performance than the $\mathrm{rGO} / \mathrm{CuNP}$ system. $\mathrm{Hu}^{65}$ and coworkers reduced the HTD by $106^{\circ} \mathrm{C}$ by the introduction of hollow mesoporous $\mathrm{CuO}$, while nanohybrids of $\mathrm{rGO} / \mathrm{CuFe}_{2} \mathrm{O}_{4}$ (ref. 66) and a new copper metal-organic framework $(\mathrm{Cu}-\mathrm{MOF})^{67}$ achieved similar results, reducing this value by $96^{\circ} \mathrm{C}$.

The increase in the energy release at $1 \%$ wt. rGO|CuNP was almost 1.8 times the energy of pure AP. In the case of $\mathrm{rGO} \mid \mathrm{CuNP}$, the increment was much important, suggesting a synergistic effect between reduced graphene oxide and the copper nanoparticles.

In accordance with the traditional electron-transfer decomposition theory of $\mathrm{AP}^{59}$ there are two controlling steps: the electron transfer from $\mathrm{ClO}_{4}{ }^{-}$to $\mathrm{NH}_{4}{ }^{+}$and the transformation from oxygen to superoxide. Therefore, graphene derivatives can accelerate the electron transfer reactions due to their high conductivity. On the other hand, partially filled orbits of semiconducting nanoparticles play a vital role as electron acceptors for the AP ions and the intermediates. ${ }^{68}$ As Fig. 7 summarizes, GO and rGO have no significant catalytic influence on the decomposition of AP. Copper nanoparticles can reduce the HTD of AP in more significant amount than CuO. During combustion of AP, CuNP are oxidized to copper oxide by the action of nitrogen oxide. ${ }^{69}$ With this oxidation, the $\mathrm{Cu}$ (II) center can act as an electron receptor of AP ions. Moreover, CuNP have larger active surface area and high-surface-to-volume ratio than CuONP and therefore, can produce higher amounts of $\mathrm{CuO}$ nanoparticles upon oxidation. The application of graphene as support of copper nanoparticles ${ }^{35}$ give better results than reduced graphene oxide. It is clear that the conjugation degree of the graphitic network affects the conductivity and thus, the presence of oxidized functional groups on the surface and edges of rGO has a direct impact on its efficiency as a catalyst. Furthermore, the functional groups of rGO can bind copper centers with higher energy than graphene, increasing the stability of copper, but diminishing its catalytic effect on the thermal decomposition of AP.

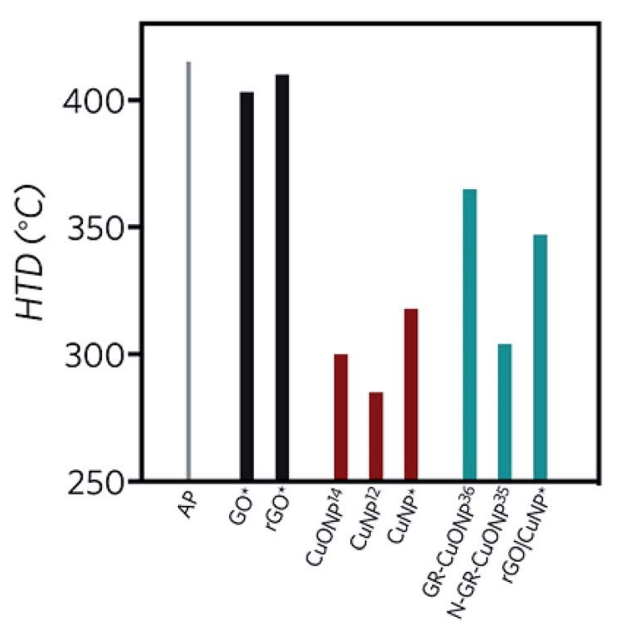

Fig. 7 Summary of the effect of GO and rGO, copper nanoparticles and supported copper nanoparticles on the HTD of AP. (*) Values obtained in this work, superscript numbers correspond to the references cited in this paper. 


\section{Conclusions}

In this work, a novel synthesis was developed to produce the in situ reduction of copper and graphene oxide by a non-toxic and environmental friendly reducing agent: L-ascorbic acid. The experimental characterization confirmed the effective reduction of copper supported on rGO. The CuNP and rGO|CuNP samples were examined by TEM, giving an average particle size of 6 and $2 \mathrm{~nm}$, respectively. The decrease of the CuNP size supported on rGO, is related to the stabilizing effect of the functional groups of rGO. Computational calculations highlighted the critical role of carbonyl and epoxy groups on the stabilization of copper nanoclusters. GO, and rGO did not affect the decomposition of AP significantly. CuNP decreased the HTD of AP by $97^{\circ} \mathrm{C}$, while rGO $\mid \mathrm{CuNP}$ diminished the HTD of AP by $68^{\circ} \mathrm{C}$. Although the HTD decreased by lesser amount in comparison to CuNP, the energy release increased 3.5 times, being higher than the CuNP catalyst and other reported works. This increase may be attributed to a synergistic effect between the reduced graphene oxide and the copper nanoparticles. The degree of conjugation of the graphitic network affects the efficiency of the catalyst. The presence of functional groups on rGO diminish the conductivity and produces higher stabilization of copper nanoparticles, increasing the necessary energy to separate the supported nanoparticles and accelerate the thermal decomposition of AP.

\section{Conflicts of interest}

There are no conflicts to declare.

\section{Acknowledgements}

M. B. C. is grateful to Fondecyt, Project Regular 1180023. C. M. V. thanks also Fondecyt Chile (Project Regular 1161297). This research was supported by the US Army W911NF-18-1-0398 and partially sponsored by the supercomputing infrastructure of the NLHPC (ECM-02). The authors are thankful to LNLSCNPEM (Brazil) and LNNano (ME-22384).

\section{References}

1 B. S. Cho and S. T. Noh, J. Appl. Polym. Sci., 2011, 121, 35603568.

2 J. Humphries, Rockets and guided missiles, Macmillan, 1956. 3 A. Davenas, J. Propul. Power, 2003, 19, 1108-1128.

4 K. K. Kuo and M. Summerfield, Fundamentals of solidpropellant combustion, American Institute of Aeronautics and Astronautics, New York, 1984.

5 J. Gao, L. Wang, Y.-l. Tai, J. Wang, J. Huo, A. M. Amin, H. Yu and W. Ding, J. Propul. Power, 2011, 27, 1143-1145.

6 P. R. Patil, V. e. N. Krishnamurthy and S. S. Joshi, Propellants, Explos., Pyrotech., 2008, 33, 266-270.

7 P. R. Patil, V. e. N. Krishnamurthy and S. S. Joshi, Propellants, Explos., Pyrotech., 2006, 31, 442-446.

8 K. Jayaraman, K. Anand, S. Chakravarthy and R. Sarathi, Combust. Flame, 2009, 156, 1662-1673.
9 S. Chaturvedi and P. N. Dave, J. Exp. Nanosci., 2012, 7, 205231.

10 S. Vyazovkin and C. A. Wight, Chem. Mater., 1999, 11, 33863393.

11 D. Survase, M. Gupta and S. Asthana, Prog. Cryst. Growth Charact. Mater., 2002, 45, 161-165.

12 L. Liu, F. Li, L. Tan, L. Ming and Y. Yi, Propellants, Explos., Pyrotech., 2004, 29, 34-38.

13 R. Dubey, P. Srivastava, I. Kapoor and G. Singh, Thermochim. Acta, 2012, 549, 102-109.

14 M. Mahinroosta, J. Nanostruct. Chem., 2013, 3, 47.

15 J. Sharma, P. Srivastava, G. Singh, M. S. Akhtar and S. Ameen, Thermochim. Acta, 2015, 614, 110-115.

16 J. L. Sabourin, D. M. Dabbs, R. A. Yetter, F. L. Dryer and I. A. Aksay, ACS Nano, 2009, 3, 3945-3954.

17 A. A. Balandin, S. Ghosh, W. Bao, I. Calizo, D. Teweldebrhan, F. Miao and C. N. Lau, Nano Lett., 2008, 8, 902-907.

18 K. S. Novoselov, A. K. Geim, S. V. Morozov, D. Jiang, M. I. Katsnelson, I. V. Grigorieva, S. V. Dubonos and A. A. Firsov, Nature, 2005, 438, 197-200.

19 S. Stankovich, D. A. Dikin, G. H. Dommett, K. M. Kohlhaas, E. J. Zimney, E. A. Stach, R. D. Piner, S. T. Nguyen and R. S. Ruoff, nature, 2006, 442, 282-286.

20 W. Cai, R. D. Piner, F. J. Stadermann, S. Park, M. A. Shaibat, Y. Ishii, D. Yang, A. Velamakanni, S. J. An and M. Stoller, Science, 2008, 321, 1815-1817.

21 S. Eigler, M. Enzelberger-Heim, S. Grimm, P. Hofmann, W. Kroener, A. Geworski, C. Dotzer, M. Röckert, J. Xiao and C. Papp, Adv. Mater., 2013, 25, 3583-3587.

22 V. López, R. S. Sundaram, C. Gómez-Navarro, D. Olea, M. Burghard, J. Gómez-Herrero, F. Zamora and K. Kern, Adv. Mater., 2009, 21, 4683-4686.

23 S. Pei and H.-M. Cheng, Carbon, 2012, 50, 3210-3228.

24 S. Stankovich, D. A. Dikin, R. D. Piner, K. A. Kohlhaas, A. Kleinhammes, Y. Jia, Y. Wu, S. T. Nguyen and R. S. Ruoff, Carbon, 2007, 45, 1558-1565.

25 D. Chen, L. Li and L. Guo, Nanotechnology, 2011, 22, 325601. 26 Z. Bo, X. Shuai, S. Mao, H. Yang, J. Qian, J. Chen, J. Yan and

K. Cen, Sci. Rep., 2014, 4, 4684.

27 C. Zhu, S. Guo, Y. Fang and S. Dong, ACS Nano, 2010, 4, 2429-2437.

28 K. De Silva, H.-H. Huang, R. Joshi and M. Yoshimura, Carbon, 2017, 119, 190-199.

29 N. Li, Z. Geng, M. Cao, L. Ren, X. Zhao, B. Liu, Y. Tian and C. Hu, Carbon, 2013, 54, 124-132.

30 Y. Yuan, W. Jiang, Y. Wang, P. Shen, F. Li, P. Li, F. Zhao and H. Gao, Appl. Surf. Sci., 2014, 303, 354-359.

31 N. A. Luechinger, E. K. Athanassiou and W. J. Stark, Nanotechnology, 2008, 19, 445201.

32 K. Zhang, Appl. Surf. Sci., 2012, 258, 7327-7329.

33 P. Fakhri, B. Jaleh and M. Nasrollahzadeh, J. Mol. Catal. A: Chem., 2014, 383, 17-22.

34 Y. Yang, Z.-H. Lu, Y. Hu, Z. Zhang, W. Shi, X. Chen and T. Wang, RSC Adv., 2014, 4, 13749-13752.

35 S. G. Hosseini, Z. Khodadadipoor and M. Mahyari, Appl. Organomet. Chem., 2018, 32, e3959. 
36 S. Paulose, R. Raghavan and B. K. George, J. Colloid Interface Sci., 2017, 494, 64-73.

37 D. C. Marcano, D. V. Kosynkin, J. M. Berlin, A. Sinitskii, Z. Sun, A. Slesarev, L. B. Alemany, W. Lu and J. M. Tour, ACS Nano, 2010, 4, 4806-4814.

38 W. S. Hummers and R. E. Offeman, J. Am. Chem. Soc., 1958, 80, 1339.

39 K. K. H. De Silva, H.-H. Huang and M. Yoshimura, Appl. Surf. Sci., 2018, 447, 338-346.

40 J. Xiong, Y. Wang, Q. Xue and X. Wu, Green Chem., 2011, 13, 900-904.

41 R. Rozada, J. I. Paredes, M. J. López, S. Villar-Rodil, I. Cabria, J. A. Alonso, A. Martínez-Alonso and J. M. Tascón, Nanoscale, 2015, 7, 2374-2390.

42 M. J. Frisch, G. W. Trucks, H. B. Schlegel, G. E. Scuseria, M. A. Robb, J. R. Cheeseman, G. Scalmani, V. Barone, G. A. Petersson, H. Nakatsuji, X. Li, M. Caricato, A. V. Marenich, J. Bloino, B. G. Janesko, R. Gomperts, B. Mennucci, H. P. Hratchian, J. V. Ortiz, A. F. Izmaylov, J. L. Sonnenberg, D. Williams-Young, F. Ding, F. Lipparini, F. Egidi, J. Goings, B. Peng, A. Petrone, T. Henderson, D. Ranasinghe, V. G. Zakrzewski, J. Gao, N. Rega, G. Zheng, W. Liang, M. Hada, M. Ehara, K. Toyota, R. Fukuda, J. Hasegawa, M. Ishida, T. Nakajima, Y. Honda, O. Kitao, H. Nakai, T. Vreven, K. Throssell, J. A. Montgomery Jr, J. E. Peralta, F. Ogliaro, M. J. Bearpark, J. J. Heyd, E. N. Brothers, K. N. Kudin, V. N. Staroverov, T. A. Keith, R. Kobayashi, J. Normand, K. Raghavachari, A. P. Rendell, J. C. Burant, S. S. Iyengar, J. Tomasi, M. Cossi, J. M. Millam, M. Klene, C. Adamo, R. Cammi, J. W. Ochterski, R. L. Martin, K. Morokuma, O. Farkas, J. B. Foresman and D. J. Fox, Gaussian 16, Revision A.03, Gaussian, Inc., Wallingford, CT, 2016.

43 A. D. Becke, J. Chem. Phys., 1993, 98, 5648.

44 C. Lee, W. Yang and R. G. Parr, Phys. Rev. B: Condens. Matter Mater. Phys., 1988, 37, 785.

45 P. J. Stephens, F. J. Devlin, C. F. Chabalowski and M. J. Frisch, J. Phys. Chem., 1994, 98, 11623.

46 P. J. Hay and W. R. Wadt, J. Chem. Phys., 1985, 82, 270.

47 A. E. Reed, R. B. Weinstock and F. Weinhold, J. Chem. Phys., 1985, 83, 735.

48 S. F. Boys and F. Bernardi, Mol. Phys., 1970, 19, 553.
49 Y. Xu, H. Bai, G. Lu, C. Li and G. Shi, J. Am. Chem. Soc., 2008, 130, 5856-5857.

50 P. Khanra, C.-N. Lee, T. Kuila, N. H. Kim, M. J. Park and J. H. Lee, Nanoscale, 2014, 6, 4864-4873.

51 Z. Luo, Y. Lu, L. A. Somers and A. C. Johnson, J. Am. Chem. Soc., 2009, 131, 898-899.

52 D. Li, M. B. Mueller, S. Gilje, R. B. Kaner and G. G. Wallace, Nat. Nanotechnol., 2008, 3, 101-105.

53 H. R. Ong, M. M. Rahman Khan, R. Ramli, Y. Du, S. Xi and R. M. Yunus, $R S C A d v$. , 2015, 5, 24544-24549.

54 N. K. Memon, A. W. McBain and S. F. Son, J. Propul. Power, 2016, 32, 682-686.

55 N. Yan, L. Qin, H. Hao, L. Hui, F. Zhao and H. Feng, Appl. Surf. Sci., 2017, 408, 51-59.

56 A. Dey, J. Athar, P. Varma, H. Prasant, A. K. Sikder and S. Chattopadhyay, RSC Adv., 2015, 5, 1950-1960.

57 J. Zhao, Z. Liu, Y. Qin and W. Hu, CrystEngComm, 2014, 16, 2001-2008.

58 R. Fitzgerald and M. Brewster, Combust. Flame, 2004, 136, 313-326.

59 H. Kumar, P. N. Tengli, V. K. Mishra, P. Tripathi, A. Bhushan and P. K. Mishra, RSC Adv., 2017, 7, 36594-36604.

60 J. Shen, Y. Hu, M. Shi, X. Lu, C. Qin, C. Li and M. Ye, Chem. Mater., 2009, 21, 3514-3520.

61 J. Nunes, D. Melo, M. Moura and R. Farias, Revista Quimica no Brasil, 2007, 1, 7-14.

62 A. P. Sanoop, R. Rajeev and B. K. George, Thermochim. Acta, 2015, 606, 34-40.

63 S. S. Joshi, P. R. Patil and V. Krishnamurthy, Def. Sci. J., 2008, 58, 721-727.

64 P. Povea, J. L. Arroyo, G. Carreño, Á. Norambuena, P. L. Rios, M. B. Camarada, I. Chavez, J. M. Manriquez and C. MoralesVerdejo, Thermochim. Acta, 2018, 666, 181-189.

65 Y. Hu, S. Yang, B. Tao, X. Liu, K. Lin, Y. Yang, R. Fan, D. Xia and D. Hao, Vacuum, 2019, 159, 105-111.

66 W. Wang and D. Zhang, RSC Adv., 2018, 8, 32221-32230.

67 S. Wang, B. Ye, C. An, J. Wang and Q. Li, J. Mater. Sci., 2019, 54, 4928-4941.

68 S. Chaturvedi and P. N. Dave, J. Saudi Chem. Soc., 2013, 17, 135-149.

69 J. Zhi and Z. Feng-Qi, J. Propul. Technol., 2002, 23, 258-261. 